\title{
Splash Formation Due to a Frog Diving into Water
}

\author{
Yoshihiro Kubota1 ${ }^{*}$, Osamu Mochizuki² \\ ${ }^{1}$ Faculty of Science and Engineering, Toyo University, Saitama, Japan \\ ${ }^{2}$ Department of Biomedical Engineering, Toyo University, Saitama, Japan \\ Email: knubota548@toyo.jp
}

Received 24 May 2015; accepted 20 July 2015; published 23 July 2015

Copyright (C) 2015 by authors and Scientific Research Publishing Inc.

This work is licensed under the Creative Commons Attribution International License (CC BY).

http://creativecommons.org/licenses/by/4.0/

(c) (i) Open Access

\begin{abstract}
Herein, we present the results of our experimental investigation of splashes formed by a frog diving into water from the ground or from a leaf and the accompanying sound generated by the impact of the frog on the water. The experiments are performed by visualizing the flow with a highspeed camera. In addition, we used physical models comprising hydrophilic bodies made from hydrogel or acrylic resin to experimentally study how hydrophilicity affects the splash. In these experiments, we use the degree of swelling to define the hydrophilicity degree. The results show that different splashes are caused by the increase in water-film velocity upon an increase in hydrophilicity. For a body with strong hydrophilicity, at a relatively high film velocity, the water film forms when the body impacts the water surface separates from the body surface. In addition, an aircavity forms when the film separates from the body. We empirically examine the relation between the hydrophilicity degree and film velocity. The results indicate that increased hydrophilicity does not reduce the splash. Therefore, we conclude that reducing of the formation of water from the biomimetic point of view is related to the shape of body.
\end{abstract}

\section{Keywords}

Frog Dive, Splash Formation, Flow Visualization

\section{Introduction}

Herein, we present an experimental investigation of the splash formed by a frog diving into water from the ground or from a leaf and the accompanying sound generated by the impact of the frog on the water. The study focuses on understanding how the hydrophilicity of a body influences splash formation and the subsequent dy-

*Corresponding author. 
namics, with the aim to understand how splash formation allows a frog to escape predators. The experiments are performed by visualizing the flow with a high-speed camera, with the sound being synchronously recorded. In addition, we experiment with physical objects of varying hydrophilicity to better understand the relation between splash formation and the hydrophilicity degree of a body.

In a related work, in 1929, Karman [1] studied the impact of a seaplane landing on a water surface [1], in which he analyzed the relation between the gliding angle and the landing speed of the seaplane. More recently, Do-Quang et al. numerically studied the water splash caused by a spherical body plunging into water [2] and found a relation between wetting phenomena, impact velocity, and splash formation. On the basis of an experimental study in which splash patters were observed for various impact velocities, Duez et al. [3] reported a relation between splashes and the accompanying sound. However, they did not discuss this phenomenon in detail. In a previous work [4], we studied the splashes formed by a solid sphere plunging into water [4] and categorized splash patterns according to impact velocity. In addition, we discussed in [4] the characteristics of the film flow generated at the early stages of the splash when a body impacted a water surface. We numerically studied the influence of strength on hydrophilicity in splash formation [5]. We computed the influence of hydrophilicity from the slip of flow.

A water droplet impinging on a solid surface also generates a splash. Yoon et al. studied the film-like structure of such splashes and its instability [6]. In a related work, Bejan et al. [7] and Bussmann et al. [8] used experimental and numerical means respectively to study the deformation of a droplet impinging on a solid surface. Bussmann et al. used the Rayleigh-Taylor instability theory to discuss the number of fingers that appeared at the edge of a spreading film. In our previous work [4], we show that instability is related to the number of droplets generated at the edge of the film flow. For a sphere plunging through a water surface, such droplets are generated by the primary splash that appears during the first stage of the splash. This work was related to that of Worthington [9], who investigated the milk crown caused by a droplet-liquid collision and the splash caused by a solid body falling into liquid milk [9]. Akers et al. [10] studied the splash of a non-Newtonian fluid [10] and showed how air cavities deform as a function of fluid properties. Ahn et al. investigated the influence of surface condition as the strength of hydrophobicity for splash erosion of soil model [11]. Finally, Truscott et al. [12] investigated the splash formed when a spinning spherical body plungeed into water, focusing particularly on the formation of underwater air cavities. Eddington et al. (2003) reported using the hydrogel as a valve for flow control on micro channels [13].

Thus, although the splash phenomenon is familiar, the detailed mechanisms behind it are not yet well understood. Because frogs dive into water often to escape predators and ensure their own survival, evolutionary reasoning suggests that their splash should be minimized. Thus, we used a high-speed camera to observe the various splashes formed by the diving of two frog species as a function of their habitat. We also recorded the sound generated by the splashes to investigate the relation between splash formation and the dive. In addition, to better understand how the hydrophilicity of a body with a hydrophilic surface (e.g., the body of a frog) affects the splash, we experimented with two homogeneous inanimate physical objects made of materials with different hydrophilicities. On the basis of these experimental results, we explain how the surface of an object affects its splash by modifying the process by which water films form. We also discuss splash formation as a function of the frog-dive technique and how hydrophilicity affects the splash and the film-flow velocity as a frog dives into water. Splashes were visualized using a high-speed CMOS camera. The tested materials are acrylic resin and a hydrogel as the hydrophilic body. These materials result in a distinctive affinity of body and water.

\section{Splash Patterns}

Figure 1 shows a tree diagram of splash patterns at different time intervals [4] [14] [15]. The evolution of the splash depends on the impact velocity. A splash is represented by the shadowed region in the figure. The three types of splashes considered are primary, secondary, and tertiary splashes. The primary splash appears first in the form of a film flow when the body touches the water surface and tiny droplets are generated from the edge of the film flow. The number of droplets can be determined by applying the instability theory to the film edge. The droplets fly out in a direction tangential to the film at the position where they detach. The secondary splash is a result of the primary splash. If the film flow follows the wall of the spherical object, then the edge of the film recombines at the top point of the object, and the secondary splash takes the form of a mushroom (see Figure 1). If the film flow does not follow the wall of the spherical object (i.e., separated flow) but the film recombines 


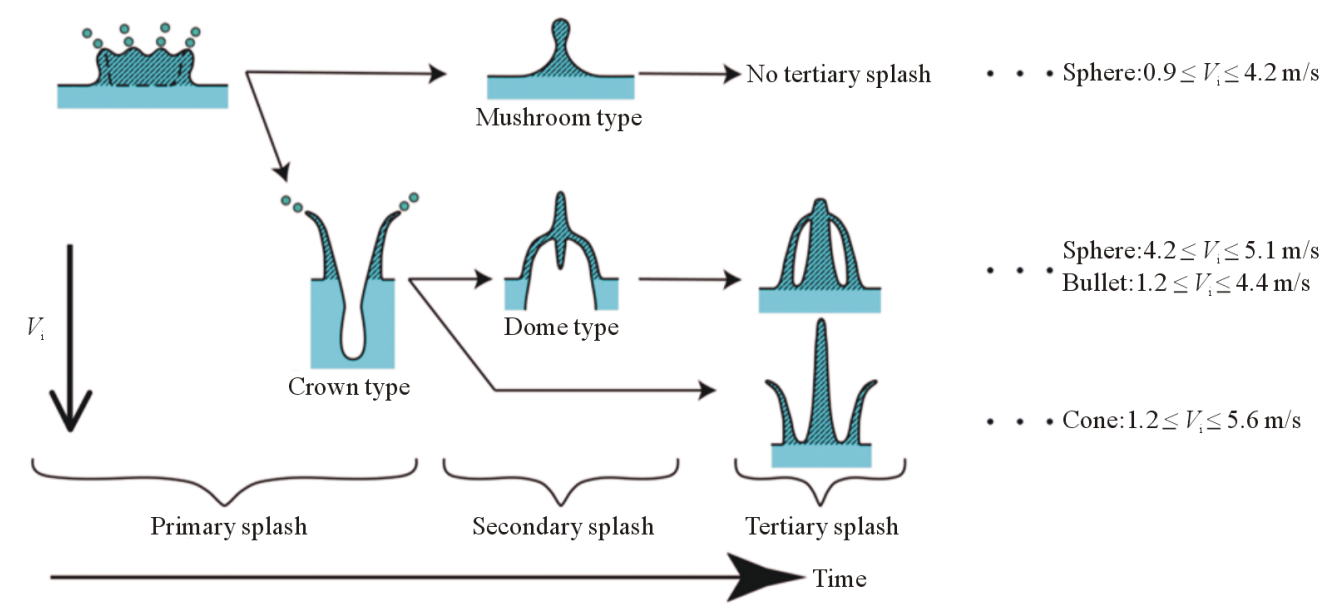

Figure 1. Tree diagram of splash formation for different impact velocities and object shapes.

above the object, the secondary splash takes the form of a dome. If the film does not recombine behind the object, no secondary splash appears. Finally, the tertiary splash appears because of the air cavity that forms when the object enters the water with sufficient speed. If no air cavity forms, no tertiary splash appears. The air cavity is related to the detachment of the film from the surface of the object. Thus, the primary splash strongly affects all subsequent splash processes.

In a previous work [14], we showed how the tail shape of the primary splash affects the secondary splash. More specifically, we reported how the shape of an object and film recombination are related to secondary-splash formation. The tail shape affects the volume of the secondary splash and the time delay between the primary and the secondary splash. We also investigated [15] how the head shape affects the formation of subsequent splash events by studying film formation as a function of the head shape. In that work, we examined the film thickness as a function of the momentum of the body and the film.

\section{Experimental Setup}

\subsection{Splash from Frog Dive}

Figure 2 shows that the sketch of experimental setup. The experiments with live frogs were performed in a water tank measuring $240 \times 200 \times 450 \mathrm{~mm}^{3}$ and filled with tap water to a depth of $100 \mathrm{~mm}$. Side-view sequential images were acquired by a high-speed CMOS camera(Vision Research Inc., Miro ex4).This camera allows 1200 images to be captured per second (1200 frames per second, or fps). A 500-W halogen lamp was used to illuminate the flow field. The sound due to the frog dives into water was recorded.

Two species of frogs, Rana porosa and Hyla japonica, were used in this experiment. R. porosa lives near flooded paddies, and H. japonica lives in trees. The different body shapes and surfaces are useful for understanding frog dives. For both frog species, the body length $L$ (i.e., from nose tip to rear of body) was $50 \mathrm{~mm}$, and the body width $W$ was $20 \mathrm{~mm}$. The initial height of the diving board was $0 \mathrm{~W}, 2.5 \mathrm{~W}$, and $5 \mathrm{~W}$, where $0 \mathrm{~W}$ means that the diving board was at the level of the water surface. The frogs' impact velocity $V_{\mathrm{i}}$ was determined by image analysis and varied from 1.7 to $2.2 \mathrm{~m} / \mathrm{s}$. The frog motion is not restricted during the experiments.

\subsection{Splash from Inanimate Objects}

To observe splashes from a solid inanimate object plunging into water, we used a water tank measuring $300 \times$ $300 \times 400 \mathrm{~mm}^{3}$ and filled it with tap water to a depth of $340 \mathrm{~mm}$. In a previous work [4], we confirmed that contaminants common to tap water do not affect splash formation. To acquire sequential side-view images of splash formation, we used a high-speed CMOS camera (Vision Research Inc., Phantom v7.1) set at 4000 fps. The each frame was exposed for $0.25 \mathrm{~ms}$. A 500- $W$ halogen lamp was used to illuminate the scene.

Spherical objects having the diameter $D=2 r=W=20 \mathrm{~mm}$ were made from acrylic resin or hydrogel. The hydrogel served as agar gel in our experiment. The wetness of each object was varied to study how the hydrophilicity degree affects the splash. We define wetness (i.e., the amount of water in the object) in terms of the swel- 


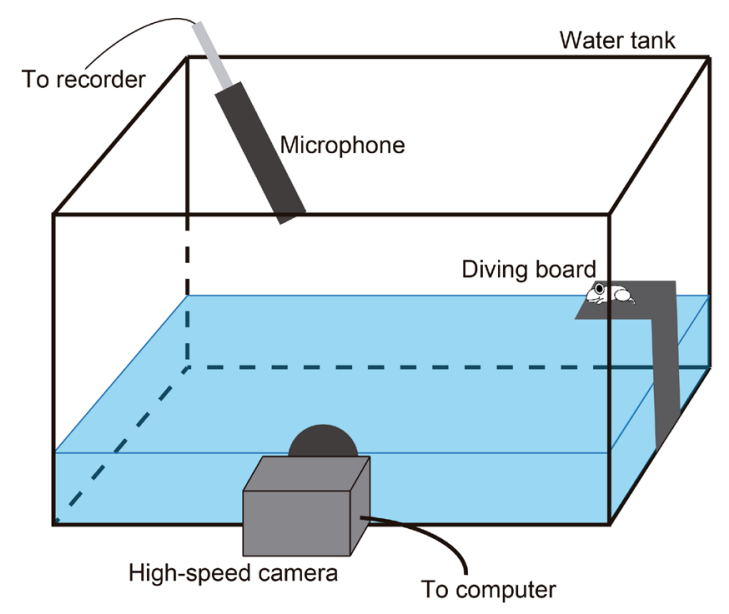

Figure 2. Sketch of experimental setup.

ling degree as follows:

$$
s=\left(m_{\text {gel }}+m_{\text {water }}\right) / m_{\text {gel }},
$$

where $m_{\text {gel }}\left(m_{\text {water }}\right)$ is the mass of gel(water). We consider swelling degrees $s=1,50$, and 100 ( $s=1$ means no water is contained in the object (e.g., an acrylic object)).

Before each experiment, we carefully wiped the surface of each object with paper towels to remove contaminants such as oil and to ensure similar surface conditions for each experiment. The impact velocity $V_{\mathrm{i}}$ of the object, which is the velocity of the object just before it enters the water, was varied by changing the height $h$ from which it was dropped. Here $h$ is defined as the distance from the water surface to the bottom of the object. The initial height $h$ was varied from $1 D$ to $45 D$, which resulted in $V_{\mathrm{i}}$ ranging from 0.63 to $4.2 \mathrm{~m} / \mathrm{s}$. The value of $V_{\mathrm{i}}$ was calculated using $V_{\mathrm{i}}=(2 g h)^{1 / 2}$, which is based on the conservation of energy. Air drag was neglected in this calculation because of the low air speed involved. A launcher system using suction held the object at the initial height before release, and all objects fell into water without rotating and with no horizontal displacement. Thus, the experiments are all repeatable.

\section{Experimental Results}

We used three dimensionless parameters to understand splash formation: dimensionless time, Reynolds number, and Weber number. The dimensionless time $T$ is defined as $T=t V_{\mathrm{i}} / D$, where $t$ is the measured time. This approach is useful for comparing how splashes form as a function of $V_{\mathrm{i}}$. Even if $V_{\mathrm{i}}$ varies, the dimensionless time $T$ $=1$ indicates the same dimensionless time interval during which the object moves a distance of one diameter $D$. The dimensionless time $T=0$ indicates the moment when the head of the objects touches the water surface. Further, Reynolds number $R e$ and Weber number We are useful for understanding the relation between the kinetics of the object and the fluid characteristics. The former is defined as $R e=V_{\mathrm{i}} D / v_{\mathrm{i}}$, where $v$ is the kinematic viscosity of water at $25^{\circ} \mathrm{C}$, and the latter is given by $W e=\rho V_{\mathrm{i}}^{2} \mathrm{D} / \sigma$, where $\rho$ is the density and $\sigma$ is the surface tension of water at $25^{\circ} \mathrm{C}$.

\subsection{Splash from Frog Dive}

Figure 3 shows consecutive images of the dive of an $R$. porosa frog into the water with an impact velocity of $V_{\mathrm{i}}$ $=1.7 \mathrm{~m} / \mathrm{s}(h / W=0)$ and the accompanying audio waveform. Figure 3(a) shows that the frog dives into the water head first. Figure 3(f) shows the final phase of the dive. The significant event in the dive is the air bubbles separating from the body. The audio waveform accompanying the dive is shown in the bottom part of Figure 3 and reveals that two significant signal bursts are generated. The first audio signal is emitted during the phenomena shown in Figure 3(a) and Figure 3(b) and corresponds to a kinematics phenomenon, namely, the sound generated when a body impacts the water surface. The second, larger-amplitude audio signal is due to bubble formation. 

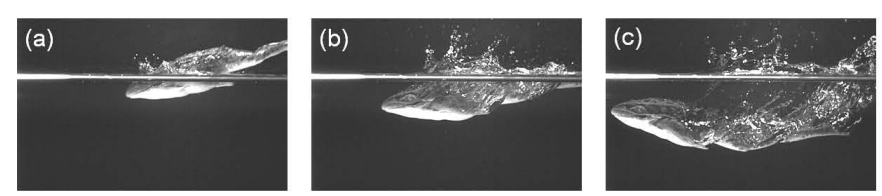

(d)

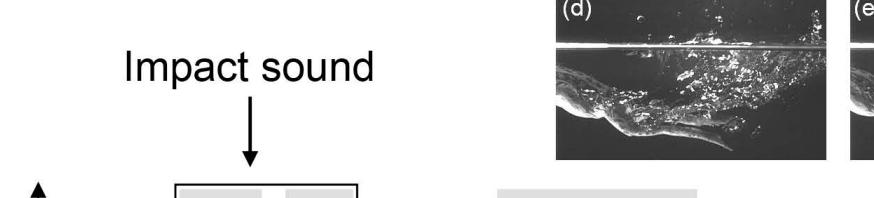

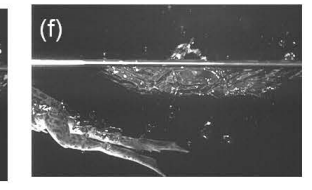

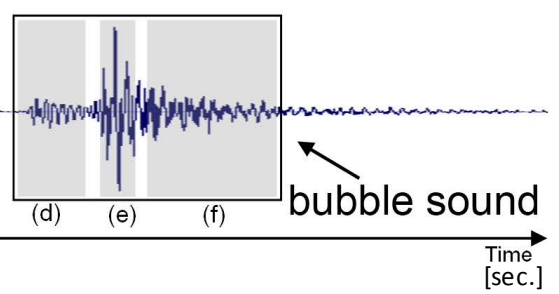

Figure 3. Images showingthe diving sequence of $R$. porosa. Shown at the bottom of the figure is the associated audio waveform.

Figure 4 shows the sequential images of the splash from the dive of an $H$. japonica frog. $H$. japonica enters the water first with its abdomen. Diving abdomen first produces a larger air cavity under the water, and the separation from the body of the cavity generates more small air bubbles than in the case of $R$. porosa. The audio wave form shown at the bottom of the figure again reveals two significant signal bursts, as in the case of the $R$. porosa dive. The initial audio burst occurs when with the body impacts the water and the second audio burst occur when the air bubbles form.

A stronger audio signal occurs at point (c) for $H$. japonica than at the corresponding point in the dive of $R$. porosa. This point corresponds to the formation of air bubbles, as seen clearly in the corresponding image. The different bubble formation for the two dives is due to the diving posture of each frog. Our previous works [12] [13] show that the shape of an object is related to the form of the air cavity created and the splash. Note that $R$. porosa lives near flooded paddies and that $H$. japonica lives in trees. This difference determines in large part how each frog escapes from its predators: $R$. porosa must escape as quietly as possible. Thus, the body posture during the dive influences the formation of air bubbles by determining at which point the air cavity separates from the body. In the next section, we use experiments with inanimate physical objects to discuss how the hydrophilicity of an object's surface affects the splash created when it plunges into water.

\subsection{Effect of Splash Formation with a Surface Condition}

The hydrophilicity degree of a body is quantified in terms of the swelling $s$, which is defined in Equation (1). Figure 5 compares the splash formed by objects having the same shape (spherical) but differing in their degree of swelling. These images correspond to the dimensionless time $T=2.0$. The acrylic body (Figure 5(a)) has $s=$ 0 because it contains no water in its body. Figure 5(b) and Figure 5(c) show the results for the hydrogel objects with $s=50$ and 100, respectively. For all three objects, a film forms when the object hits the water surface and the droplets are generated at the edge of the film. The splash for $s=0$ is of the mushroom type, where the film recombines behind the object. A mushroom-type splash forms when no underwater air cavity forms. The splash for the hydrogel for $s=50$ and 100 is of the dome type. The dome-type splash forms when the body completely penetrates the water surface before the film covers the body. Note that the depth of the air cavity differs for $s=$ 50 and $s=100$. This depth is greater for greater hydrophilicity (i.e., for $s=100$ ), which means that the hydrophilicity degree of an object's surface affects the velocity of film flow. To better understand the relation between film velocity and swelling, we measured the film velocity as a function of swelling.

Figure 5(a) shows, as a function of $V_{\mathrm{i}}$, the maximum film velocity $V_{\text {fmax }}$ measured at $T=0.05$ and for $s=0$ (red circles). For comparison, the maximum film velocity for $s=50$ and 100 was measured at $V_{\mathrm{i}}=3.1 \mathrm{~m} / \mathrm{s}$ (triangle, square, and cross). From the result for $s=0$, we obtain $V_{\text {fmax }}=8.3 V_{\mathrm{i}}$. The maximum film velocity for the hydrophilic object at $V_{\mathrm{i}}=3.1 \mathrm{~m} / \mathrm{s}$ is more than twice that for $s=0$ (i.e., acrylic object), which we attribute to the 

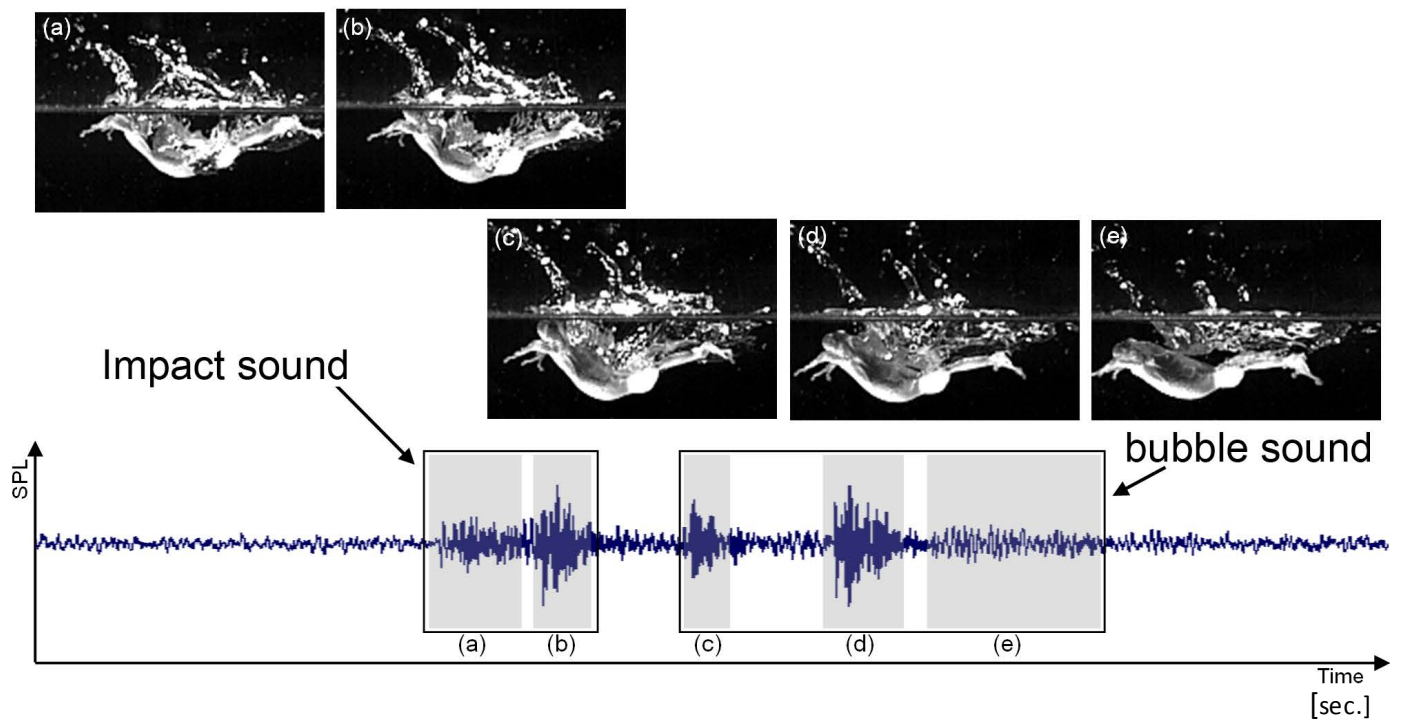

Figure 4. Images showingthe diving sequence of $H$. japonica. Shown at the bottom of the figure is the associated audio wave form.

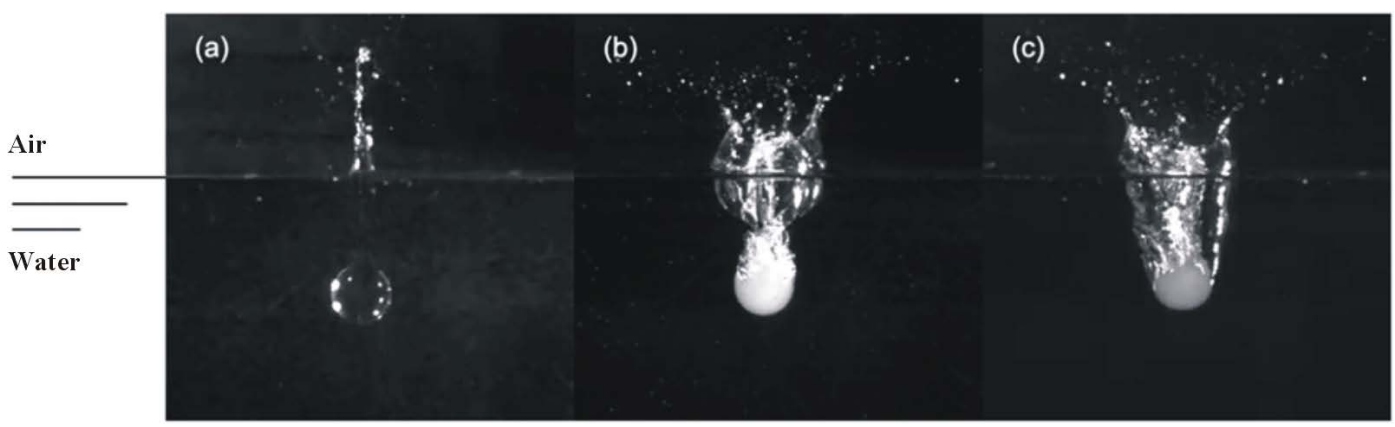

Figure 5. Comparison of splash formation at $V_{\mathrm{i}}=3.1 \mathrm{~m} / \mathrm{s}$. (a) $s=0$ (acrylic resin); (b) $s=50$; and (c) $s=100$.

film separating from the surface of the object [4]. The film separates when the centrifugal force acting on the film becomes greater than the surface tension of the film [4] [15]. The film velocity increases with swelling, which indicates that hydrophilicity is related to splash formation. Thus, we need to understand the relation between the hydrophilicity degree and splash formation.

Figure 6(b) shows the relation between film velocity and swelling. The red circles show the results of experiments, and the blue line shows the empirical function $V_{\mathrm{fmax}}=0.98 \ln (s)$. The empirical function is obtained on the basis of the evidence shown in Figure 5 that film velocity increases with hydrophilicity. Figure 5 also reveals that the depth of the air cavity increases with an increase in swelling, which means that amore hydrophilic surface leads to a deeper air cavity. Thus, the air cavity must be considered in addition to the impact velocity to determine the potential energy of the system and the film velocity. Therefore, a combination of impact velocity and film velocity affects the tertiary splash, because the tertiary splash forms from the air cavity. In addition, the empirical curve is consistent with the experimental data. Thus, the film velocity of a hydrophilic object can be expressed as a logarithmic function of the swelling of the object.

If the impact velocity is less than $0.9 \mathrm{~m} / \mathrm{s}$, then a spire-type splash forms because of the concavity of the water surface [4]. From the relation between film velocity and impact velocity, we infer that the film velocity at a low impact velocity increases. This means that the impact-velocity threshold between spire- and mushroom-type splashes $\left(V_{\mathrm{i}}<0.9 \mathrm{~m} / \mathrm{s}\right.$ at $\left.s=0\right)$ changes as a function of the hydrophilicity of an object's surface. To confirm the increase in film velocity, we visualize the splash. Figure 7(a) shows that aspire-type splash forms for $s=0$, and Figure 7(b) shows the result for $s=50$. The spire-type splash in Figure 7(a) formed because of a reaction to the concavity of the film. No spire-type splash is seen in Figure 7(b), because the film does not separate from the 

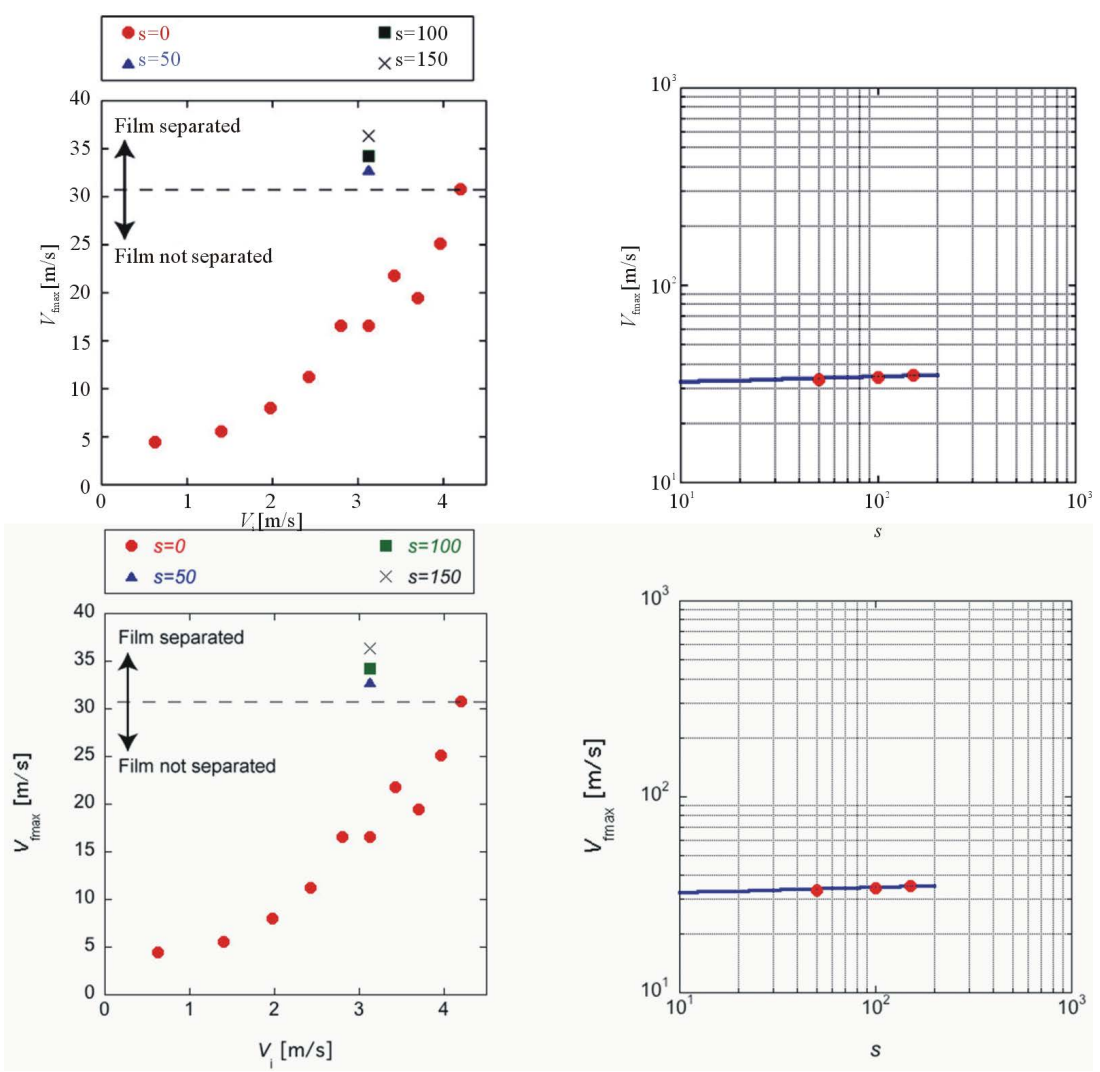

Figure 6. Effect of hydrophilicity on film velocity.

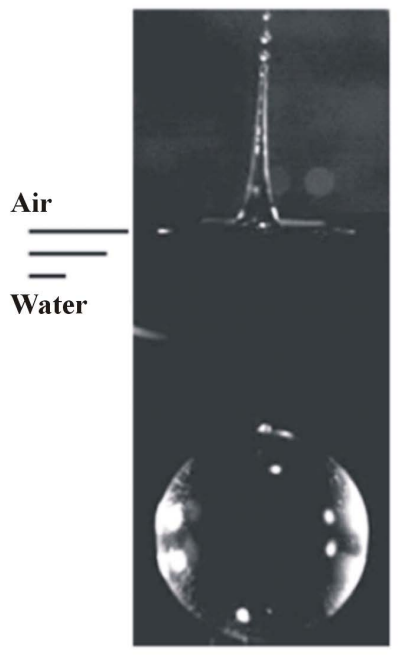

(a)

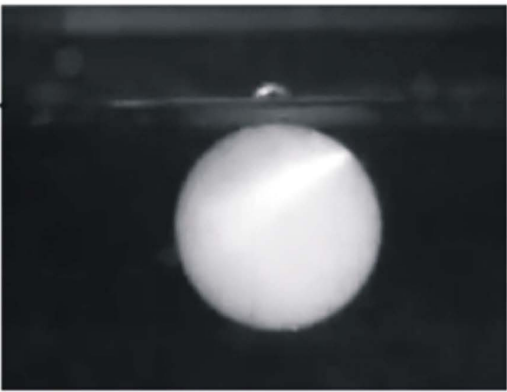

(b)

Figure 7. Splash for $V_{\mathrm{i}}=0.63 \mathrm{~m} / \mathrm{s}$ and (a) $s=0$ (acrylic object), and (b) $s=50$ (hydrophilic object).

object before the concavity forms. This result indicates the importance of understanding the relation between film velocity and impact velocity.

\section{Concluding Remarks}

We observed splash formation for a frog diving into water and for an inanimate solid object falling into water. 
The splash for the frog dive was caused by the impact of the frog's body and by the reaction to the separation of the air cavity from the body. The sound generated by the frog dive was strongly related to the formation of air bubbles, which was due to the collapse of the air cavity.

We used a high-speed CMOS camera to observe the splash of a hydrophilic object. The results showed that an object's hydrophilicity affects splash formation. Further, the hydrophilicity degree varied with swelling. We studied splashes from swelling $s=0,50$, and 100, where swelling indicated the amount of water inside the object ( $s$ $=0$ for the acrylic object). To study the effect of hydrophilicity, we used objects made of hydrogel. Changing of the objects' surface conditions allowed us to study how they affect the film flow.

The results showed that the secondary splash depended on the hydrophilicity and on whether the film recombines behind the object. The acrylic object $(s=0)$ creates a mushroom-type splash, and the hydrogel objects with $s=50$ and 100 createdome-type splashes. Whether a mushroom-type splash or a dome-type splash occurrence was determined by whether the film separated from the surface of the object. Film separation led to the formation of an air cavity, which was related to the formation of the tertiary splash. Thus, the hydrogel objects generated an additional splash in the form of a tertiary splash.

The film separated because of excessive film velocity. We obtained an empirical logarithmic expression for film velocity as a function of swelling. Thus, hydrophilicity affected film velocity, which in turn determined whether the film separated from the object's surface. Film separation led to a tertiary splash.

By comparing the results of the frog dive with those of the experiment, we found that the significant bubble entrainment was caused by $H$. japonica, which revealed the importance of shape for experiments on splash formation. In other words, the hydrophilic characteristic of the surface of an object affected splash formation. The splash caused by $H$. japonica would thus be reduced when it adopted an appropriate body position.

\section{References}

[1] von Karman, T. (1929) The Impact on Seaplane Floats during Landing. National Advisory Committee for Aeronautics Technical, Note 321.

[2] Minh, D.-Q. and Amberg, G. (2009) The Splash of a Solid Sphere Impacting on a Liquid Surface: Numerical Simulation of the Influence of Wetting. Physics of Fluid, 21, Article ID: 022102. http://dx.doi.org/10.1063/1.3073968

[3] Duez, C.Y., Clanet, C. and Bocquet, L. (2007) Making a Splash with Water Repellency. Nature Physics, 3, 180-183. http://dx.doi.org/10.1038/nphys545

[4] Kubota, Y. and Mochizuki, O. (2009) Splash Formation by a Spherical Body Plunging into Water. Journal of Visualization, 12, 339-345. http://dx.doi.org/10.1007/BF03181877

[5] Yokoyama, M., Kubota, Y., Kikuchi, K., Yagawa, G. and Mochizuki, O. (2014) Some Remarks on Surface Conditions of Solid Body Plunging into Water with Particle Method. Advanced Modeling and Simulation in Engineering Sciences, 1, 1-14. http://dx.doi.org/10.1186/2213-7467-1-9

[6] Yoon, S.S., Jepsen, R.A., Nissen, M.R. and O’Hern, T.J. (2007) Experimental Investigation on Splashing and Nonlinear Fingerlike Instability of Large Water Drops. Journal of Fluids and Structure, 23, 101-115. http://dx.doi.org/10.1016/j.jfluidstructs.2006.08.009

[7] Bejan, A. and Gobin, D. (2006) Constructal Theory of Droplet Impact Theory. International Journal of Heat and Mass Transfer, 49, 2412-2419. http://dx.doi.org/10.1016/j.ijheatmasstransfer.2006.02.001

[8] Bussmann, M., Chandra, S. and Mostaghimi, J. (2000) Modeling the Splash of a Droplet Impacting a Solid Surface. Physics of Fluids, 12, 3121-3132. http://dx.doi.org/10.1063/1.1321258

[9] Worthington, A.M. (1882) On Impact with a Liquid Surface. Proceedings of the Royal Society of London, 34, 217-230. http://dx.doi.org/10.1098/rspl.1882.0035

[10] Akers, B. and Belmote, A. (2006) Impact Dynamics of a Solid Sphere Falling into a Viscoelastic Micellar Fluid. Journal of Non-Newtonian Fluid Mechanics, 135, 97-108. http://dx.doi.org/10.1016/j.jnnfm.2006.01.004

[11] Ahn, S., Doer, S.H., Douglas, P., Bryant, R., Hamlett, C.A.E., McHale, G., Newton, M.I. and Shircliffe, N.J. (2013) Effects of Hydrophobicity on Splash Erosion of Model Soil Particles by a Single Water Drop Impact. Earth Surface Processes and Landforms, 38, 1225-1233. http://dx.doi.org/10.1002/esp.3364

[12] Truscott, T.T. and Techt, A.H. (2006) Cavity Formation in the Wake of Spinning of Sphere Impacting the Free Surface. Physic of Fluids, 18, Article ID: 091113. http://dx.doi.org/10.1063/1.2335903

[13] Eddington, D.T. and Beebe, D.J. (2004) Flow Control with Hydrogels. Advanced Drug Delivery Reviews, 56, $199-210$. http://dx.doi.org/10.1016/j.addr.2003.08.013 
[14] Kubota, Y. and Mochizuki, O. (2010) Elemental Structure of Splash Generated by a Plunging Solid Body. Journal of Flow Visualization and Image Processing, 17, 359-369. http://dx.doi.org/10.1615/JFlowVisImageProc.v17.i4.70

[15] Kubota, Y. and Mochizuki, O. (2011) Influence of Head Shape of Solid Body Plunging into Water on Splash Formation. Journal of Visualization, 14, 111-119. http://dx.doi.org/10.1007/s12650-011-0071-4 